\title{
Stability of Oxygen-enriched Nanoclusters and Helium Bubbles in Fe-based Alloys under Extreme Conditions
}

\author{
M. K. Miller, C. L. Fu, Xinqiu Chen*, and Q. Li \\ Materials Science and Technology Division, Oak Ridge National Laboratory, Oak Ridge, TN \\ 37831 USA \\ *Currently at Shenyang National Laboratory for Materials Sciences, PR China
}

Stable nanoclusters (NCs) have been observed in Fe-based alloys fabricated by mechanical alloying [1]. The 2-4-nm-diameter Ti-Y-O-enriched NCs in these nanostructured ferritic alloys (14YWT) not only maintain remarkable stability up to 0.92 of the melting temperature and under high dose irradiation conditions, but also display outstanding creep properties. Nanophase materials are known to be metastable in nature, because of coarsening processes that occur rapidly at elevated temperatures. However, these NCs are an exception to this norm.

First-principles theory has shown that the fundamental feature that differentiates these NCs from equilibrium compound phases is that vacancies play an indispensable role in their structure and stability [2]. Most compound phases can accommodate vacancies on the lattice sites under equilibrium conditions; however, the concentration of vacancies is usually a few parts per million. By contrast, the vacancy concentration in these NCs is extremely high, and the binding between vacancies and interstitial $\mathrm{O}$ atoms is central to their structure and stability. These NCs are in a new form of a defective alloying state [2,3]. Furthermore, the NCs have a disordered structure and the NC size is limited by their exceptionally stable interface [3].

Atom probe tomography (APT), scanning transmission electron microscopy (STEM), and in-situ small angle neutron scattering (SANS) experiments have investigated the formation of NCs in mechanically-alloyed 14YWT powder after isothermal annealing. APT and SANS confirmed that NCs form after short term annealing. Consistent with the first-principles results, STEM imaging indicates that the NCs lack structural order [4]. Measurements by positron lifetime spectroscopy have provided evidence for the presence of vacancy-related features in NCcontaining alloys [5].

Nanocluster-strengthened alloys are more resistant to He-induced embrittlement during neutron irradiation than reduced activation/ferritic martensitic steels. Recent APT and TEM studies revealed that $\mathrm{He}$ bubbles are preferentially formed in the close vicinity of the $\mathrm{NC} / \mathrm{matrix}$ interfaces, as shown in Figure 1. The distribution of He bubbles in a He-implanted 14YWT material indicate that $\sim 48.6 \%$ of the He bubbles are located on NCs, $4.4 \%$ on other precipitates, $\sim 14.4 \%$ on grain boundaries, $\sim 12.2 \%$ on dislocations, and the remainder as isolated bubbles in the matrix [6].

Density functional calculations indicated that He interstitials in Fe tend to bind both to other He atoms and vacancies, resulting in stable He-vacancy complexes. Although the vacancies are in the form of O-vacancy pairs in the NCs, we find that an O-vacancy pair is still able to bind with multiple He interstitials. The binding energy of a $n^{\text {th }}$ He atom attached to a [(n-1)He + vacancy] cluster or a $[(n-1) \mathrm{He}+\mathrm{O}$-vacancy pair] cluster in the Fe matrix as a function of the number of 
helium atoms is shown in Figure 2. Without $\mathrm{O}$, a vacancy can attract up to eight He atoms. In the presence of $\mathrm{O}$-vacancy pair, the number of the He atoms that can be attached to an oxygenvacancy pair is reduced to four. Although these small [(O-vacancy) $+\mathrm{He}]$ clusters represent nascent He bubbles, the presence of $\mathrm{O}$ limits the size of the helium clusters. The calculation suggests that, as observed by $\mathrm{APT}$, the $\mathrm{NC} /$ matrix interface regions are the preferential nucleation/trapping sites for $\mathrm{He}$ bubbles (and thereby reduce the susceptibility to $\mathrm{He}$ embrittlement) due to the existence of a high O-vacancy pair concentration in the NCs.

\section{References:}

[1] MK Miller et al, Front. Mater. Sci. China 3(1) (2009) p. 9.

[2] CL Fu et al, Physical Review Letters 99 (2007) 225502.

[3] H Zhao et al, Physical Review B84 (2011) 144115.

[4] MC Brandes et al, J. Mater. Sci. 47 (2012) p. 3913.

[5] J Xu, et al, Phys. Rev. B, 79 (2009) 020204.

[6] PD Edmondson, et al, J. Nucl. Mater 434 (2013) p. 210.

[7] Research supported by the U.S. Department of Energy (DOE), Basic Energy Sciences (BES), Materials Sciences and Engineering Division, and through a user project supported by ORNL's Shared Research Equipment (ShaRE) User Program, also sponsored by DOE-BES.
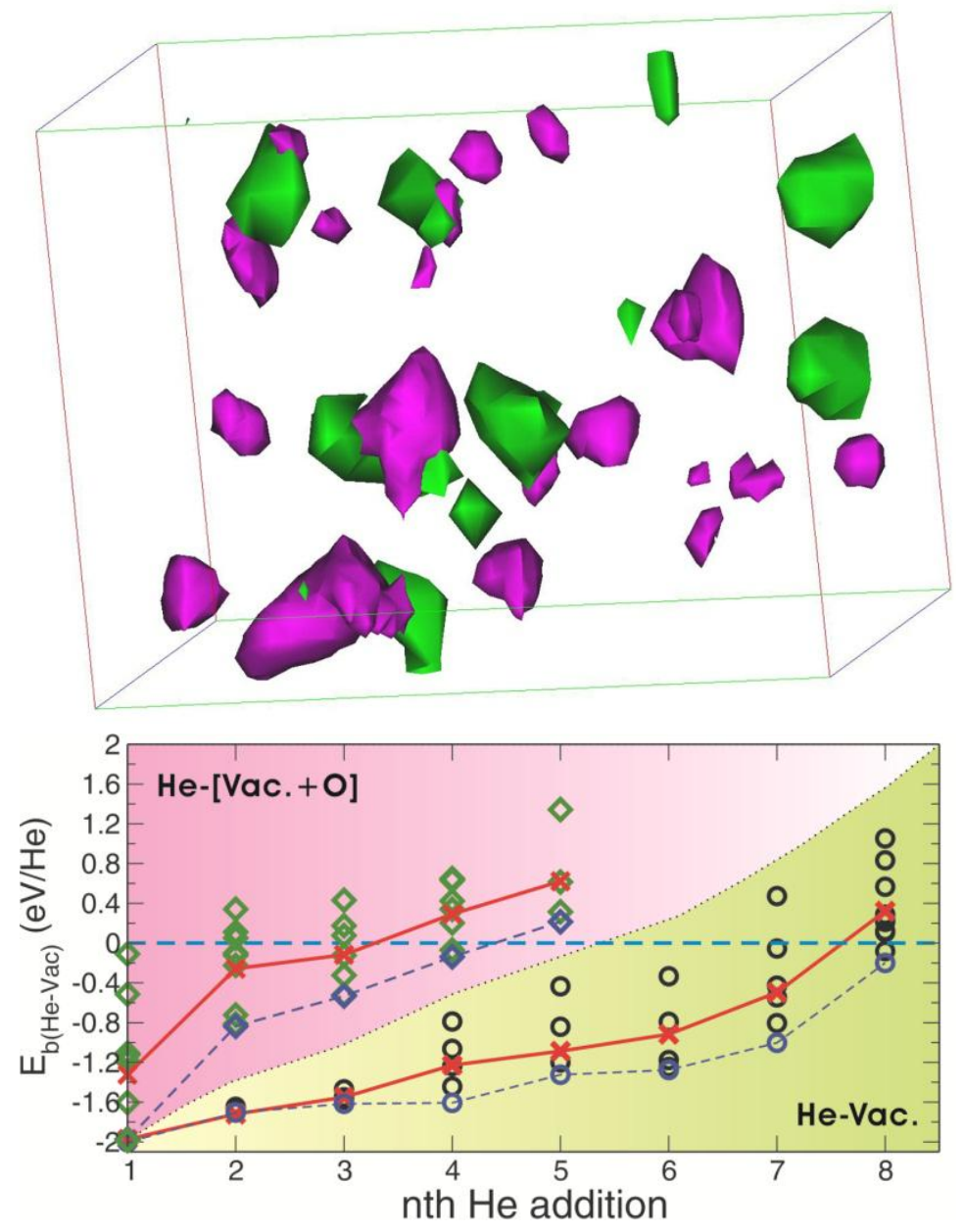

Figure 1. 2\% $\mathrm{Ti}$ isoconcentration (green) and $75 \%$ isodensity (purple) surfaces showing the distribution of NCs and He bubbles in 14YWT NFA that was He implanted to a dose of $6.75 \times 10^{16}$

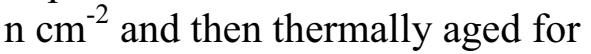
$100 \mathrm{~h}$ at $750^{\circ} \mathrm{C}$. Volume $40 \times 30 \mathrm{x}$ $22 \mathrm{~nm}$.

Figure 2. The binding energy of a $\mathrm{n}^{\text {th }}$ helium atom $\left(\mathrm{E}_{\mathrm{b}}\right)$ attached to clusters containing a vacancy and (n-1) He atoms (lower curves) and an oxygen-vacancy pair and (n-1) He atoms (upper curves). The solid and dashed lines represent the average and lowest binding energies, respectively, among all atomic configurations being considered. As the binding energy becomes positive, the addition of the $n^{\text {th }} \mathrm{He}$ atom is detached from the cluster. 